\title{
Editorial, Applied Practices Special Issue
}

As Editor of this Special Issue of Applied Practices in the Journal of Psychologists and Counsellors in Schools, I am delighted to introduce 10 great articles to you all. The Special Issue is a combination of six invited applied papers from leading Australian researchers, which I will overview and summarise in some detail, and four practitioner papers. Asking already busy top researchers to write something new and relevant for the profession was potentially challenging. I wish to thank all the invited persons for their generosity in first saying yes and then following through across several months in preparing these specific applied papers. Most Australian states are represented by an author, with a fascinating range of topics.

Every article draws out the realities of applying research findings when working as a practitioner in schools. For simplicity I will use the nomenclature 'school counsellor' here, noting that this term is inclusive of school counsellors, guidance officers and school psychologists. Challenges in writing for practitioners in Australia are both the different titles used and the different models of working as a school counsellor in each state or territory. Nevertheless, every article offers key strategies about effective working practices, as well as providing important clear information about the approach they are presenting. Key themes across all the invited papers include evidence-based research, student involvement, working as part of a school team and with the whole school, overcoming barriers to successful school counselling work, positive and strengths-based approaches, and the specific skills brought by the school counsellor when engaging with students, their families and their teachers in individual counselling and intervention work. In presenting the processes necessary to apply evidence-based practices in schools, the invited papers offer a model to school counsellors about how to effectively implement initiatives in schools.

Professor Donna Cross and her team have developed Friendly Schools, a systematic approach to bullying, over many years. In her article, 'Friendly Schools Bullying Prevention Research: Implications for School Counsellors', she and her co-authors summarise relevant key findings from a large range of research on bullying, with a focus on the role of school counsellors. The links between bullying and ongoing mental health difficulties are acknowledged, making the enhancement of protective factors, such as parent-child relationships, and early prevention of and intervention with bullying, of crucial importance. The section on bullying perpetrators raises some interesting specific factors and interventions needing to be explored further, as perpetrators might be resistant to typical interventions. School counsellors' wide-ranging contributions as part of a whole school team are emphasised, including their specific role in providing confidential safe support for students experiencing bullying either as a victim or perpetrator or both.

A second article exploring bullying, particularly cyberbullying, is from Professor Barbara Spears and colleagues, titled 'Using Participatory Design to Inform Cyber/Bullying Prevention and Intervention Practices: Evidence-Informed Insights and Strategies'. The foci on student voice, as well as school staff voice and participation, are fascinating and are highlighted in the two exemplar case studies. School counsellors' prevention and intervention work with others is acknowledged, while also stressing the importance of context and specificity. COVID 19 pandemic factors are included and integrated meaningfully. Personally, I found the sections contextualising children and young people, historically and in relation to their rights and participation, particularly interesting and helpful when considering bullying and other issues impacting students in schools and beyond in 2021. The detailed case studies can be 
viewed as models for school counsellors about how to work comprehensively within the school team, and how to involve students, including younger children.

Dr John Burns and Professor Ron Rapee explore the critical topic of universal screening for mental health issues in schools. 'From Barriers to Implementation: Advancing Universal Mental Health Screening in Schools' focuses on screening; however, interestingly, the four stages outlined could be applied to other initiatives that school counsellors may want to action in school contexts. The article focuses on key internalising mental health issues, such as anxiety, depression and mood disorders. These problems tend to be both amenable to a screening program, especially as they may be less obvious than externalising behaviour problems, and of crucial importance in terms of further assessment, counselling support and potential targeted interventions.

'Social Emotional Learning in the Early Years: Innovation and Applications' is an excellent applied paper from Associate Professor Erica Frydenberg. Erica is well known for her extensive work on coping, both in developing assessment tools and providing interventions to ensure better and more productive coping. In this article she reports on varied, interesting and systematic applied research processes followed over a decade with trainee educational psychologists, linking social-emotional learning in the early years with coping and resilience in children ranging in age from 3 to 8 years. Coping is examined using novel approaches for young children, with linked data from their families, leading to interventions through parenting programs, including with culturally different parents.

'Positive Education: The Key Role of the School Counsellor', by Dr Suzy Green and her colleague Kasey Lloyd, examines the major role a school counsellor can play in ensuring a wellbeing-oriented approach using Positive Education (and Positive Psychology) principles in school settings. In common with other papers, the wider inclusion of school counsellors in implementing new approaches is acknowledged, with barriers stated as well as realistic guidelines being clearly outlined. The authors outline the links between students' wellbeing and academic progress, as well as exploring the role of schools in ensuring optimal wellbeing functioning in students, alongside schools' roles in achieving educational goals. I found the case study presented of a school counsellor undertaking the key roles needed in implementing Positive Education enhanced my understanding of the range of processes needed to bring about positive change.

The final invited paper is from Professor Matt Sanders and his team. In 'Delivering Evidence-Based Parenting Support in Educational Settings', the crucial factors of effective parenting are outlined, and the role of schools in overtly supporting parents is presented in detail. Many educators talk up parentschool links and cooperation to facilitate students' learning, without following through by implementing truly supportive and potentially fruitful partnerships. I found the sections providing a framework of support for parental self-regulation fascinating and nonjudgmental. All parents (and educators) can learn the skills of self-regulation and ensure these skills are passed on to children. Many such skills begin during the early years, prior to children entering formal education; however, positive parenting, as outlined in the TripleP parenting programs, can be facilitated in school contexts, with new programs described that specifically target teacher-parent relationships. School counsellors are ideally placed to liaise between families and schools, and to ensure programs supporting parents to gain optimal parenting skills are actioned. The positive impact of parenting programs in schools is clear in the article, with a large body of evidence-based programs presented. Ultimately, good parenting can make classrooms better learning and wellbeing environments, and help students become balanced contributors to their society.

Four practitioner papers are also part of this Special Issue. Daisuke Ito and colleagues present an exploratory pilot study in a Japanese high school on the effect of a mindfulness program on adolescents who have been traumatised. The advantages of a school context in introducing a mental health initiative are stressed, with the school counsellor taking a lead role in the intervention. Mindfulness practice was combined with psychoeducation and applications of mindfulness to the students' presenting problems. Further, teachers were asked to encourage and reinforce students' application of the skills and strategies, which is a potential enhancing feature in schools if managed well. The promising results suggest further research in this area is warranted 
Rachel Yerbury's article, titled “'Let's be Still”: A School Psychologist Delivered Stillness Meditation Program for Wellbeing', addresses a related approach with a preventative program, using stillness meditation. This program is based around a specially written book read across 10 weeks and designed for young students (7- to 9-year-olds). Core outcomes, using thematic analyses, was in the 'voices' of the children and their teachers across five school years with different young students. Common positive descriptors from the children were 'calm', 'free', 'happy', and 'peaceful', with teachers finding the impact of the program 'advantageous'.

'Therapeutic Crisis Intervention in Schools (TCI-S): An International Exploration of a Therapeutic Framework' to reduce critical incidents and improve teacher and student emotional competence in schools is a descriptive and analytic paper from Stella Rogers and Sharinaz Hassan, focused on the TCI-S approach, which is a whole school framework for managing and reducing critical incidents. As a trauma-informed framework, TCI-S is applicable in dealing with problem behaviours in school contexts. To date, the work has mostly been in specialised schools but with the underlying principles having wider potential application.

In the final article in this Special Issue, Dr John Burns, who co-authored one of the invited papers, provides an excellent practitioner paper titled 'Towards Best Practice in School Management of Students with Depressive Disorders'. He offers a well-structured overview of this key topic for school counsellors working in school settings. Universal mental health promotion, preventative approaches, treatment and, importantly, maintenance are all examined, with their key impacts clearly articulated. Although John's focus is on depression, the principal areas would be applicable to other prevalent and pervasive mental health issues, such as anxiety.

I am certain as readers you will all enjoy this Special Issue of the journal, focused on applied papers with direct relevance to your work and with examples from the field. It has been a wonderful opportunity to invite and receive the six papers from leading researchers, and to accompany these with four excellent practitioner papers. If I had to provide a short overview title it would be 'Actioning Research Within Schools: Key Processes for Successful Working'. 